\title{
Aspergillus Species in Lower Respiratory Tract of Hospitalized Patients from Shanghai, China: Species Diversity and Emerging Azole Resistance
}

This article was published in the following Dove Press journal: Infection and Drug Resistance

\author{
Yuan $X u^{1,2}$ \\ Min Chen ${ }^{2}$ \\ Junhao Zhu ${ }^{3}$ \\ Bert Gerrits van den Ende 4 \\ Amanda Juan $\mathrm{Chen}^{5}$ \\ Abdullah MS Al-Hatmi ${ }^{6,7}$ \\ $\mathrm{Li} \mathrm{Li}^{3}$ \\ Qiangqiang Zhang ${ }^{3}$ \\ Jianping $\mathrm{Xu}$ iD ${ }^{8}$ \\ Wanqing Liao ${ }^{2}$ \\ Yuchong Chen'
}

'Department of Dermatology, The Third People's Hospital of Hangzhou, Hangzhou, People's Republic of China; ${ }^{2}$ Department of Dermatology, Shanghai Key Laboratory of Molecular Medical Mycology, ChangZheng Hospital, Second Military Medical University, Shanghai, People's Republic of China; ${ }^{3}$ Department of Dermatology, Huashan Hospital, Fudan University, Shanghai, People's Republic of China; ${ }^{4}$ Department of Food and Indoor Mycology, Westerdijk Fungal Biodiversity Institute, Utrecht, the Netherlands; ${ }^{5}$ Department of Medical Mycology, Institute of Medicinal Plant Development, Chinese Academy of Medical Sciences and Peking Union Medical College, Beijing, People's Republic of China; ${ }^{6}$ Centre of Expertise in Mycology, Radboud University Medical Centre, Canisius Wilhelmina Hospital, Nijmegen, the Netherlands; ${ }^{7}$ Ministry of Health,

Directorate General of Health Services, Ibri, Oman; ${ }^{8}$ Department of Biology, McMaster University, Hamilton, Canada; ${ }^{9}$ Department of Dermatosurgery, Shanghai Skin Diseases Hospital, School of Medicine, Tongii University, Shanghai, People's Republic of China

Correspondence: Yuchong Chen $\mathrm{Tel} / \mathrm{Fax}+86-2 \mathrm{I}-6 \mathrm{I} 833148$

Email cyuchong@aliyun.com

Junhao Zhu

Tel/Fax +86-2I-8I 885502

Email zhujh1985@I26.com
Purpose: To investigate species diversity and prevalence of antifungal resistance among clinical isolates of Aspergillus spp. in Shanghai, China.

Patients and Methods: In this study, the Aspergillus spp. isolates were analyzed by multilocus sequence typing (MLST) targeting the internal transcribed spacer (ITS) regions, and partial $\beta$-tubulin $(B e n A)$ and calmodulin $(\mathrm{CaM})$ genes. The susceptibilities of these isolates to nine antifungal agents were determined according to the protocol in document M38-A3 established by the Clinical and Laboratory Standards Institute (CLSI).

Results: The most common Aspergillus spp. was A. fumigatus (58.2\%), followed by the A. flavus complex (23.5\%), and A. niger complex (15.3\%). Isolates belonging to A. tamarii and A. effusus of the A. flavus complex and A. tubingensis and A. awamori of the A. niger complex were identified. Moreover, several mutations were found in the azole target cyp51A gene $\left(\mathrm{TR}_{46} / \mathrm{Y} 121 \mathrm{~F} / \mathrm{T} 289 \mathrm{~A}\right.$ and F46Y, G89G, M172V, N248T and D255E) in azole-resistant isolates of A. fumigatus.

Conclusion: The results of our study revealed a diversity of species in the lower respiratory tract of inpatients in Shanghai and approximately $9 \%$ of our isolates were resistant to at least one of the triazole antifungals. Formulation of local treatment strategies to combat emerging azole resistance and species diversity in clinically relevant Aspergillus spp. is needed.

Keywords: Aspergillus, antifungal susceptibility, identification, molecular typing, China

\section{Introduction}

Aspergillus is a diverse genus with a high economic, social and health impact, in which about 40 Aspergillus spp. are clinically relevant. ${ }^{1-3}$ The $A$. fumigatus complex is the major cause of aspergillosis worldwide, followed by the A. flavus complex, the A. niger complex, and A. terreus. ${ }^{1,2,4}$ Aspergillus spp. can cause a broad spectrum of pulmonary aspergillosis (PA), ranging from an allergic reaction (allergic bronchopulmonary aspergillosis; ABPA) to various infections such as chronic pulmonary aspergillosis (CPA) or invasive pulmonary aspergillosis (IPA).$^{5-7}$ Aspergillus spp. colonization is an important prerequisite to subsequent infections, ${ }^{8}$ particularly in the lower respiratory airways. ${ }^{8,9}$

The differentiation of Aspergillus spp. at the species level has been shown to correlate with specific patterns of antifungal susceptibility. ${ }^{7,10,11}$ However, morphological features are frequently insufficient to differentiate Aspergillus spp. to species level. ${ }^{1,4,12}$ Over the last decade, molecular identification methods, such as multilocus sequence typing (MLST) targeting the internal transcribed spacer (ITS), partial 
$\beta$-tubulin (BenA) and calmodulin ( $\mathrm{CaM})$ gene regions, have shown capable of discriminating cryptic species among morphologically similar Aspergillus spp. ${ }^{1,13}$ Consequently, epidemiological characteristics of clinical isolates of Aspergillus spp. have become increasingly accurate. ${ }^{7,14,15}$ For example, several non-A. fumigatus spp. and cryptic species within the genus Aspergillus, have been reported as causative agents of aspergillosis worldwide, including China. ${ }^{16-18}$

The recommended first-line drugs to treat aspergillosis are triazoles. ${ }^{19}$ However, triazole-resistant isolates of Aspergillus spp. have been increasingly detected across the globe since the early 2000s, likely due to the widespread use of azoles in agriculture and clinics. ${ }^{15,20,21}$ Several resistance mechanisms have been reported in A. fumigatus isolates, ${ }^{21}$ particularly mutations in the cyp $51 \mathrm{~A}$ gene. ${ }^{22}$ The main resistance mechanism, $\mathrm{TR}_{34} / \mathrm{L} 98 \mathrm{H}$, has been documented since 2007. ${ }^{23}$ More recently, an emerging mutation, $\mathrm{TR}_{46} / \mathrm{Y} 121 \mathrm{~F} /$ T289A, has been identified in many European countries. ${ }^{22,24}$ In China, while the majority of mutations are the $\mathrm{TR}_{34} / \mathrm{L} 98 \mathrm{H}$, new mutations, such as $\mathrm{TR}_{46} / \mathrm{Y} 121 \mathrm{~F} / \mathrm{T} 289 \mathrm{~A}$, have been identified frequently since 2010 . $^{18,25}$

Geographical differences in the prevalence of different Aspergillus spp., especially in azole-resistant A. fumigatus have been observed among national and regional populations in the world, including China. ${ }^{26,27}$ Although several studies on A. fumigatus in China have been reported, ${ }^{26,28}$ studies focused on isolates of non- $A$. fumigatus spp. are limited, especially on cryptic Aspergillus species. Moreover, antifungal resistance in these reported isolates is rare. ${ }^{8,18,29}$

Shanghai, the largest and a medically well-developed city located in Eastern China, is currently faced with an increasing number of hospitalized patients coming from not only Shanghai but also other parts of China. Thus, Aspergillus infections of the hospitalized patients in Shanghai could provide a snapshot of the national profile on aspergillosis. The aim of this study was to investigate the diversity of Aspergillus spp. isolated from the lower respiratory airways of hospitalized patients in Shanghai via an MLST method and to determine their in vitro susceptibility to currently available antifungal drugs.

\section{Patients and Methods Collection of Clinical Isolates}

From January 2016 to March 2018, a total of 98 isolates of Aspergillus spp. were collected from the bronchoalveolar lavage fluid (BALF) belonging to 98 hospitalized patients.
The patients were identified to have proven or probable Aspergillus spp. infections or colonization prior to their admissions to two tertiary teaching hospitals: Shanghai Changzheng Hospital (1280-beds) and Shanghai Huashan Hospital (1216-beds). Ethical approval was obtained, and all patients involved understood and agreed to the usage of the clinical isolates in the present study. The details of the isolates are listed in Supplementary Table S1.

\section{Isolation and Phenotypic Identification}

The putative isolates of Aspergillus spp. were first grown on Sabouraud dextrose agar (SDA; Oxoid, UK) for three days at $35^{\circ} \mathrm{C}$ in aerobic conditions. These isolates were then morphologically identified as Aspergillus spp. using Czapek-dox agar (CDA; Oxoid, UK) and potato dextrose agar (PDA; Oxoid, UK) according to their macromorphological and microscopic features of the colonies. ${ }^{30}$

\section{Molecular Identification}

Genomic DNA of each isolate was extracted following the CTAB protocol described previously. ${ }^{31}$ Fragments of the three genes BenA, CaM, and ITS regions were amplified directly from the genomic DNA as described previously. ${ }^{1,32,33}$ PCR products were sequenced on an ABI 3770XL capillary sequencer (Applied Biosystems, Lennik, The Netherlands). Sequence reads were assembled and edited using SeqMan v.7.0.0 (DNASTAR, Madison, WI, USA). The details of the primer sequence and PCR amplification conditions are listed in Supplementary Table S2.

The sequences were aligned using the server version of the MAFFTv. 7.0 (www.ebi.ac.uk/Tools/msa/mafft/), followed by the manual checking in BIOEDIT v. 7.0.5.2. All sequences of the isolates have been deposited in GenBank, and the accession numbers are listed in Supplementary Table S1. Representative sequences from known species of the Aspergillus genus were retrieved from GenBank and included in our alignment. Corresponding gene sequences of Penicillium chrysogenum strain CBS 306.48 were chosen as outgroup in phylogenetic analysis. The best-fit model of sequence evolution was determined by MEGA version 7.0.14 (Center for Evolutionary Medicine and Informatics, Tempe, AZ). After verifying the best models, phylogenetic trees were inferred using the maximum likelihood (ML) method with 1000 rounds of re-sampling, and bootstrap branch support $>80 \%$ was regarded as robust for species identification. Phylogenetic trees were viewed and edited with FIGTREE v. 1.1.2 software. 


\section{In vitro Antifungal Susceptibility}

According to the criteria of M38-A3 in document established by Clinical and Laboratory Standards Institute (CLSI), ${ }^{34}$ we determined in vitro susceptibility of all isolates to itraconazole (ITZ, Sigma-Aldrich, Basingstoke, UK), voriconazole (VRZ, Sigma-Aldrich, Basingstoke, UK), posaconazole (PSZ, Sigma-Aldrich, Basingstoke, UK), ravuconazole (RVZ, Toronto Research Chemicals Inc, Toronto, Canada), isavuconazole (ISZ, Toronto Research Chemicals Inc, Toronto, Canada), anidulafungin (AFG, Toronto Research Chemicals Inc, Toronto, Canada), caspofungin (CFG, Sigma-Aldrich, Basingstoke, UK), micafungin (MFG, Toronto Research Chemicals Inc, Toronto, Canada), and amphotericin B (AmB, Sigma-Aldrich, Basingstoke, UK). Final concentrations of antifungal agents ranged from 0.03 to $16 \mu \mathrm{g} / \mathrm{mL}$ for ITZ, VRZ, PSZ, RVZ, ISZ and AmB, and 0.015 to $8 \mu \mathrm{g} / \mathrm{mL}$ for AFG, CFG and MFG. Stock solutions of drugs were prepared in dimethyl sulfoxide, and stored at $80^{\circ} \mathrm{C}$ until used. The reference isolate used here for comparison was Candida parapsilosis strain ATCC 22,019. The in vitro susceptibility testing was performed in triplicates for each isolate. The Minimal inhibitory concentration (MIC) data obtained were reported as the ranges, $\mathrm{MIC}_{50}$ and $\mathrm{MIC}_{90}$. The proposed epidemiological cutoff values (ECVs) of Aspergillus spp. for ITZ, VRZ, PSZ, ISZ, CFG and AmB followed those by the CLSI for antifungal agents (Supplementary Table S3) ${ }^{35-38}$ There are no ECVs currently available for RVZ, AFG or MFG.

\section{Sequencing of $A$. fumigatus cyp5 I A Gene}

The full sequences of the cyp51A gene, including the promoter region, of all triazole-resistant $A$. fumigatus isolates were amplified and sequenced following the protocols described previously. ${ }^{39}$ The sequences obtained were aligned with the sequence of a triazole-susceptible isolate (GenBank accession AF338659) using ClustalW software. ${ }^{40}$ Specifically, we followed the steps described by Deng et $\mathrm{al}^{28}$ and scanned the predicted cyp51A aminoacid sequence for substitutions, particularly those linked to triazole resistance as identified previously.

\section{Statistical Analysis}

The data collected during the study period were analyzed using IBM SPSS Statistics 23.0 software. Percentages of the species diversity among different patient populations were compared using $\chi^{2}$ test. A $P$ value less than 0.05 was considered statistically significant.

\section{Results}

\section{Demographic Data of the Patients}

In our study, the mean age of the patients was 61.9 ( \pm standard deviation: 16.6 years; range: 18-94 years), with the largest group in the age range of $61-80$ years $(48.0 \%$; 47/98). The male/female gender ratio was $3.1(74 / 24)$. According to the recently defined standardized criteria, ${ }^{7}$ the clinical profiles of the patients were divided into colonization (80.6\%; 79/98), CPA $(14.3 \%$; 14/98), IPA (3.1\%; $3 / 98)$, and ABPA $(2.0 \% ; 2 / 98)$ groups. No significant differences $(P$ value $=0.642)$ were observed among the age group of the patients about the species diversity (Figure 1). Remarkably, the proportion of A. fumigatus among the colonization patients $(53.2 \%$; $42 / 79)$ was significantly lower $(P$ value $=0.034)$ than that among the diseased patients with clinical aspergillosis (CPA, IPA and ABPA) $(78.9 \% ; 15 / 19)$. All the patients were HIVnegative, whereas half of them had underlying conditions. The most prevalent underlying condition was type 2 diabetes mellitus $(15.3 \%$; 15/98), followed by solid tumor (12.2\%; 12/98), chronic lung disease $(10.2 \% ; 10 / 98)$, and chronic liver disease $(5.1 \% ; 5 / 98)$, etc. About 15\% (15/98) of the patients had more than one kind of underlying condition. The detailed clinical profiles of each patient are presented in Supplementary Table S1.

\section{Identification of Aspergillus spp}

A total of six species complex were unambiguously identified among the 98 isolates based on sequences of three DNA markers, including A. fumigatus (58.2\%; 57/98), the A. flavus complex $(23.5 \% ; 23 / 98)$, the $A$. niger complex $(15.3 \% ; 15 / 98)$, A. sydowii $(1.0 \% ; 1 / 98)$, A. terreus $(1.0 \% ; 1 / 98)$, and A. nidulans $(1.0 \% ; 1 / 98)$. Interestingly, our phylogenetic analysis based on the concatenated sequences (BenA, CaM and ITS) identified four rarely observed species in these patients, A. tamarii and A. effusus in the A. flavus complex; and A. tubingensis and A. awamori in the A. niger complex (Figure 2 and Supplementary Table S1).

\section{In vitro Antifungal Drug Susceptibility}

With the exception of azoles and AmB in some cases, most tested antifungal compounds demonstrated potent activity against the 98 Aspergillus isolates (Table 1). The antifungal drug echinocandins exhibited the MIC values ranging from 0.015 to $1 \mu \mathrm{g} / \mathrm{mL}$. AmB also showed potent activity, with MICs ranging from 1 to $4.0 \mu \mathrm{g} / \mathrm{mL}$, and with geometric means (GM) closer to the lowest MIC value, which was 

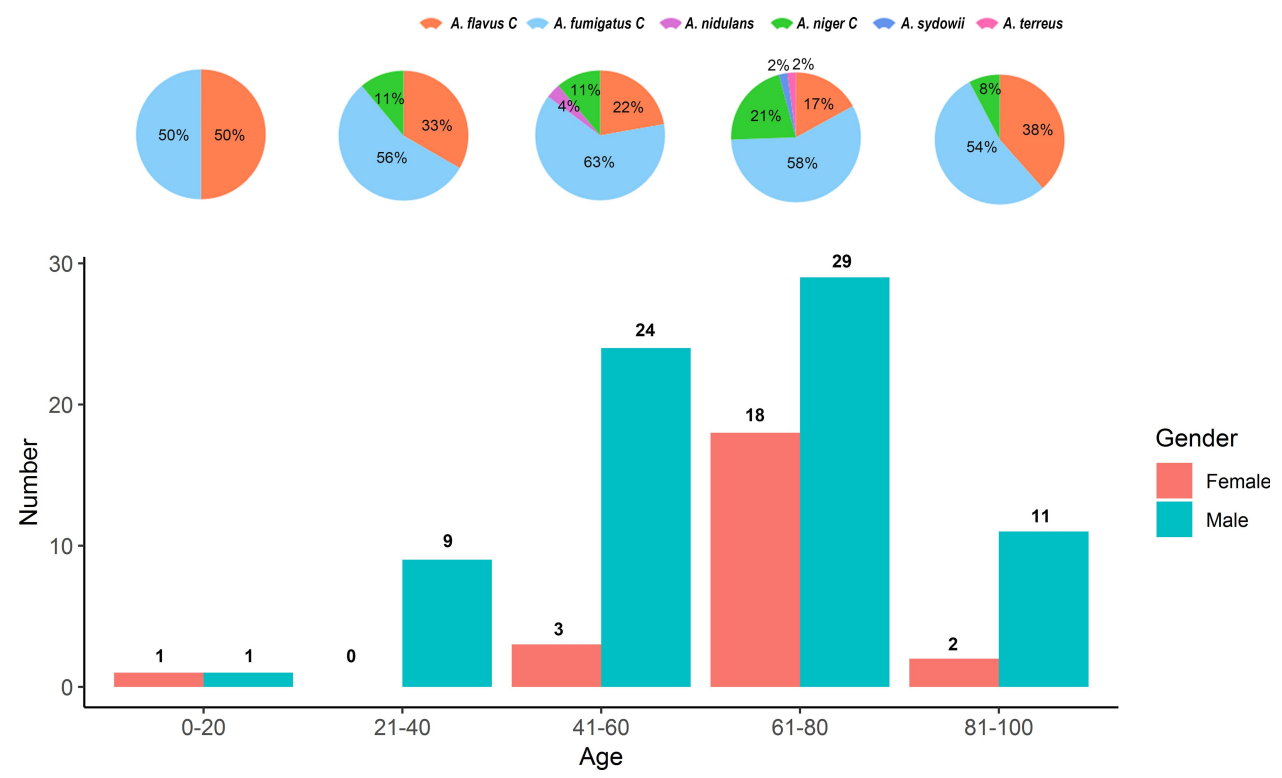

Figure I Distribution of Aspergillus spp. in the lower respiratory tract of hospitalized patients of different ages and sexes in Shanghai, China. (The top pie chart shows Aspergillus species distribution within each of the five 20-year age groups, $0-20 ; 21-40 ; 4 I-60 ; 6 I-80$; and $8 I-100$. The bottom bar graph shows the number of Aspergillus isolates from the two sexes for each of the five age groups. Aside from the $0-20$ age group where only two isolates were obtained, $A$. fumigatus was the most common species across all age groups and that more isolates were obtained from males than females).

observed against $A$. fumigatus $(1.245 \mu \mathrm{g} / \mathrm{mL})$, the $A$. flavus complex $(1.620 \mu \mathrm{g} / \mathrm{mL})$ and the $A$. niger complex $(1.05 \mu \mathrm{g} /$ $\mathrm{mL})$. For azoles, more variable results were observed with MIC values ranging from 0.06 to $16.0 \mu \mathrm{g} / \mathrm{mL}$. The lowest azole GM MIC value was PSZ with GM MIC (0.187 $\mu \mathrm{g} /$ $\mathrm{mL}$ ) against $A$. fumigatus, and the highest was ISZ against A. niger, with GM MIC value $(2.297 \mu \mathrm{g} / \mathrm{mL})$. Of the 98 isolates, 11 showed resistance to one or several of the tested antifungal drugs (Table 2). Among the 57 isolates of A. fumigatus, four were resistant to the azoles, with one isolate (in particular CMXY 13,113) being resistant to several azoles ITZ, VRZ, RVZ and ISZ. Two A. fumigatus isolates (CMXY 14,287 and CMXY 10,234) were resistant to AmB. Among the 23 isolates of the A. flavus complex, four were resistant to azoles, while two isolates (CMXY 22,879 and CMXY 27,481) were resistant to selected azoles (PSZ and ISZ) and AmB. One A. tubingensis isolate (CMXY 27,207) was resistant to multiple antifungals ITZ, VRZ, RVZ, PSZ and ISZ.

\section{Sequence Variation Among Triazole-Resistant Isolates at the cyp5 IA Gene}

Among the azole-resistant strains of A. fumigatus, one (CMXY13,113) had two mutations in the cyp51A gene and a 46-bp tandem repeat in the gene promoter $\left(\mathrm{TR}_{46}\right.$ /Y121F/T289A). One ITZ resistant strain (CMXY15837) and one PSZ and ISA double resistance strain (CMXY28940) of $A$. fumigatus showed several polymorphisms in the cyp51A gene (F46Y, G89G, M172V, N248T, $\mathrm{D} 255 \mathrm{E})$. However, no cyp51A promoter or amino-acid sequence variation was observed for the azole-resistant isolate CMXY25241 (Table 2).

\section{Discussion}

Aspergillus spp. cause a wide spectrum of human diseases and affect more than 14 million people worldwide.,41 Diverse Aspergillus spp. frequently differ in their susceptibilities to antifungal drugs and geographical differences exist in the prevalence of different Aspergillus spp. ${ }^{42,43}$ The results of our study expand the knowledge on species diversity and antifungal susceptibility of Aspergillus spp. from hospitalized patients in Shanghai, China.

In our study, colonization was the dominant (80.6\%) clinical profile of Aspergillus spp. among the hospitalized patients, which should be taken seriously in China. Several publications indicate colonization to be an important risk factor for the development of IPA, ${ }^{8,44}$ with approximately $18 \%$ of colonized patients developing IPA, especially patients with COPD. ${ }^{45}$ The proportion of the patients colonized by non- $A$. fumigatus is significantly higher than that among the patients with CPA, IPA or ABPA, which was not observed in a similar study reported from Japan. ${ }^{9}$ The significance of non- $A$. fumigatus, especially 


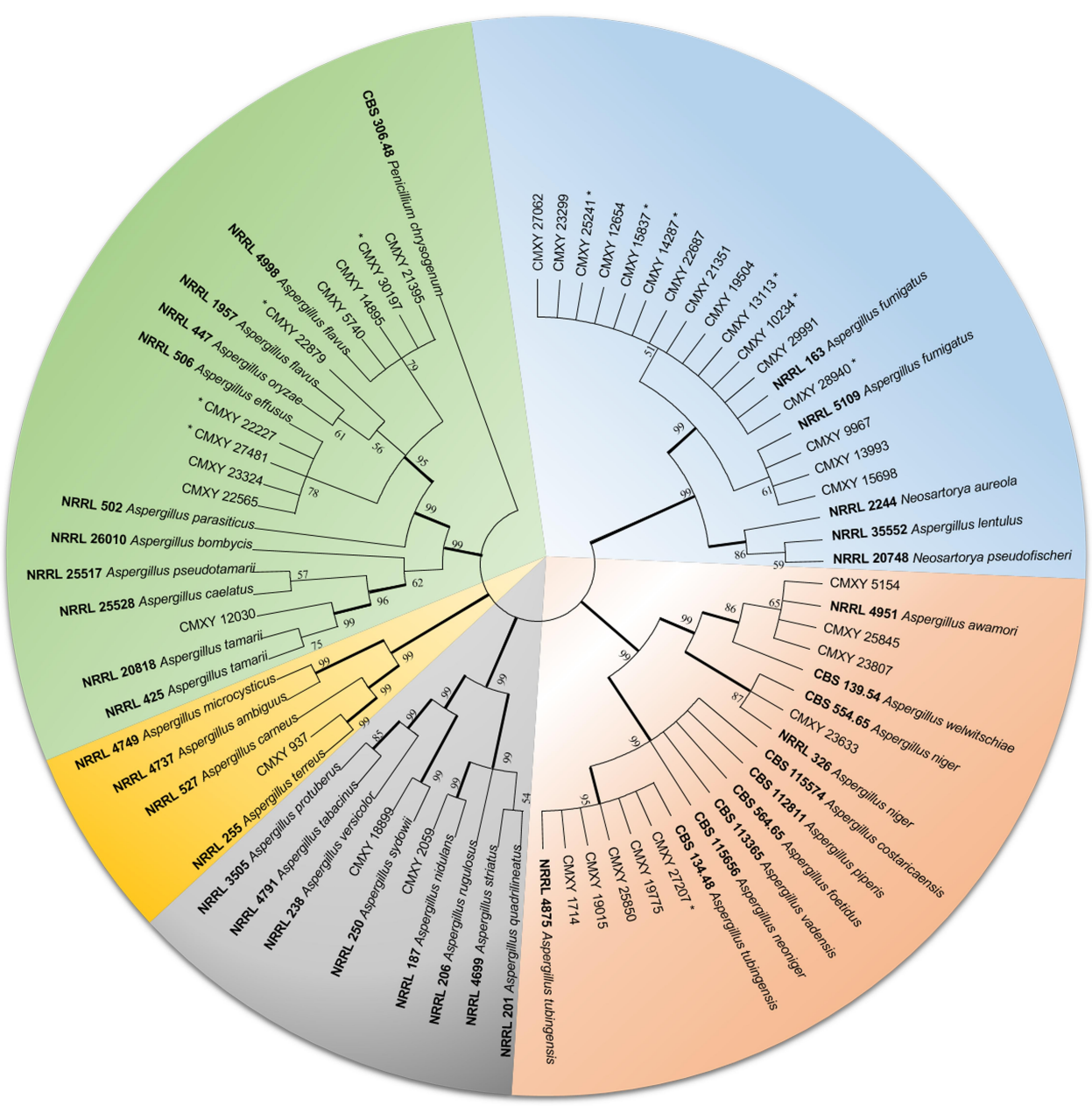

Figure 2 A phylogenetic tree for the representative Aspergillus spp. isolates from our study using the maximum likelihood method based on the combined sequences of ITS, BenA and CaM loci. (Type strains for all species found in our samples were included as references for confirmation of species identification. "*”" means antifungal resistant isolates of Aspergillus spp. in our study; "bold font" indicates type strain for that species).

the $A$. niger complex, colonizing the lower respiratory airways among immunocompromised patients, needs further research. ${ }^{8} \mathrm{CPA}$ is the second frequent profile of Aspergillus spp. in our study, though the exact incidence is unclear in other regions. ${ }^{7}$ The prevalence of IPA (3.1\%) in our study was within the range reported previously in Europe $(0.2-6.9 \%) .^{46,47}$ Similarly, the prevalence of ABPA $(2 \%)$ in our sample was similar to the recent data from a global survey $(2.5 \%){ }^{48,49}$

In the present study, A. fumigatus was the most frequently isolated $(58.2 \%)$. This species is known to be prevalent in indoor environments in $\mathrm{China}^{50,51}$ and has a superior ability to survive in the lower respiratory tracts of humans. ${ }^{7}$ Similar prevalence has also been reported from Beijing, China $(59.3 \%)^{18}$ and Madrid, Spain $(54.7 \%) .{ }^{52}$ The A. flavus complex is the second (23.5\%) most common group of Aspergillus spp. in our study, which is also the second most prevalent etiological agent of IA worldwide. ${ }^{18,53}$ It should be noted that the A. flavus complex has been reported to cause outbreaks in hospital, especially after surgery in high-risk patients. ${ }^{53}$ In India, the $A$. flavus species complex was also the most common Aspergillus spp. isolated from air-conditioned areas. $^{54}$ Although the prevalence of IA caused by the A. niger complex has increased recently, ${ }^{55}$ all isolates of the $A$. niger complex analyzed in this study were collected from colonized patients but without active aspergillosis. However, one patient had diabetes and the prognosis of pulmonary colonization caused by the $A$. niger complex is generally poor among patients with diabetes. ${ }^{56}$ In addition, we identified one $A$. sydowii isolate from lower respiratory tract of a female patient. This species also is an important etiologic agent of superficial infections. $^{57}$ 
Table I Summary Distribution of Susceptibilities of the 98 Clinical Isolates of Aspergillus spp. to Nine Antifungal Drugs as Determined Based on the M38-3A Protocol Established by the Clinical and Laboratory Standards Institute (CLSI)

\begin{tabular}{|c|c|c|c|c|c|c|c|c|c|c|}
\hline \multirow[t]{2}{*}{ Species/Number } & & \multicolumn{9}{|c|}{ MIC or MEC $(\mu g / m L)$ for } \\
\hline & & AFG & CFG & MFG & ITZ & VRZ & RVZ & PSZ & ISZ & AmB \\
\hline \multirow{4}{*}{$\begin{array}{l}\text { A. fumigatus complex } \\
\text { (57) }\end{array}$} & MIC range & $\leq 0.015-0.03$ & $0.06-1$ & $\leq 0.015-0.12$ & $0.25-8$ & $0.12-16$ & $0.25-16$ & $0.06-1$ & $0.25-16$ & $1-4$ \\
\hline & $\mathrm{MIC}_{50}$ & $\leq 0.015$ & 0.25 & 0.03 & 0.5 & 0.5 & 1 & 0.25 & & 1 \\
\hline & $\mathrm{MIC}_{90}$ & 0.03 & 0.25 & 0.12 & 1 & 0.5 & 2 & 0.25 & 1 & 2 \\
\hline & GM & 0.021 & 0.221 & 0.034 & 0.615 & 0.443 & 0.765 & 0.187 & 0.711 & 1.245 \\
\hline \multirow[t]{4}{*}{ A. flavus complex (23) } & MIC range & $\leq 0.015-0.25$ & $0.12-0.25$ & $\leq 0.015-0.25$ & $0.12-0.5$ & $0.5-2$ & $\mathrm{I}-2$ & $0.12-1$ & $0.5-2$ & $1-4$ \\
\hline & $\mathrm{MIC}_{50}$ & 0.03 & 0.12 & 0.06 & 0.25 & I & 1 & 0.25 & 1 & 2 \\
\hline & $\mathrm{MIC}_{90}$ & 0.06 & 0.25 & 0.12 & 0.5 & 2 & 2 & 1 & & 2 \\
\hline & GM & 0.034 & 0.169 & 0.057 & 0.291 & 0.970 & 1.128 & 0.328 & 1.031 & 1.620 \\
\hline \multirow[t]{4}{*}{ A. niger complex (I5) } & MIC range & $\leq 0.015-0.03$ & $0.06-0.12$ & $0.03-0.25$ & $0.5-16$ & $0.5-16$ & $0.5-16$ & $0.25-2$ & $0.5-16$ & $0.5-2$ \\
\hline & $\mathrm{MIC}_{50}$ & $\leq 0.015$ & 0.12 & 0.12 & 1 & 1 & 2 & 0.5 & 2 & 1 \\
\hline & $\mathrm{MIC}_{90}$ & 0.03 & 0.12 & 0.25 & 1 & 2 & 4 & 0.5 & & 2 \\
\hline & GM & 0.020 & 0.109 & 0.090 & 0.912 & 1.260 & 1.910 & 0.416 & 2.297 & 1.05 \\
\hline A. sydowii (I) & MIC range & 0.06 & 0.03 & 0.03 & I & 2 & 2 & 1 & 2 & 2 \\
\hline A. terreus (I) & MIC range & $\leq 0.015$ & 0.12 & $\leq 0.015$ & 0.25 & 2 & 2 & 0.12 & 1 & 2 \\
\hline A. nidulans (I) & MIC range & 0.06 & 0.06 & 0.03 & 0.5 & 0.25 & 0.25 & 0.06 & 0.25 & 2 \\
\hline
\end{tabular}

Abbreviations: AFG, anidulafungin; CFG, caspofungin; MFG, micafungin; ITZ, itraconazole; VRZ, voriconazole; RVZ, ravuconazole; PSZ, posaconazole; ISZ, isavuconazole; $\mathrm{AmB}$, amphotericin B; GM, geometric means; MIC, Minimal inhibitory concentration; MEC, Minimal effective concentration; MIC 50 , MIC/MEC at which $50 \%$ of the isolates tested were inhibited; MIC $_{90}$, MIC/MEC at which $90 \%$ of the isolates tested were inhibited.

Table 2 Minimal Inhibitory Concentrations of Antifungal Drug-Resistant Isolates of Aspergillus spp. in Our Study as Determined Based on the M38-3A Protocol Established by the Clinical and Laboratory Standards Institute (CLSI)

\begin{tabular}{|c|c|c|c|c|c|c|c|c|}
\hline \multirow[t]{2}{*}{ Species } & \multirow[t]{2}{*}{ Number } & \multicolumn{6}{|c|}{ MIC $(\mu \mathrm{g} / \mathrm{mL})$ for } & \multirow[t]{2}{*}{ cyp5IA } \\
\hline & & ITZ & VRZ & $\mathbf{R V Z}$ & PSZ & ISZ & AmB & \\
\hline A. fumigatus & CMXY 13,113 & 8 & 16 & 16 & I & 16 & $\dagger$ & $\mathrm{TR}_{46} / \mathrm{Y} 12 \mathrm{IF} / \mathrm{T} 289 \mathrm{~A}$ \\
\hline A. fumigatus & CMXY I5,837 & 4 & $\dagger$ & $\dagger$ & $\dagger$ & $\dagger$ & $\dagger$ & F46Y, G89G, MI72V, N248T, D255E \\
\hline A. fumigatus & CMXY 28,940 & $\dagger$ & $\dagger$ & $t$ & 0.5 & 2 & $t$ & F46Y, G89G, MI72V, N248T, D255E \\
\hline A. fumigatus & CMXY 25,241 & $\dagger$ & $\dagger$ & $t$ & I & $\dagger$ & $\dagger$ & \# \\
\hline A. fumigatus & CMXY 10,234 & $t$ & $\dagger$ & $t$ & $\dagger$ & $\dagger$ & 4 & \# \\
\hline A. fumigatus & CMXY 14,287 & $\dagger$ & $\dagger$ & $t$ & $\dagger$ & $\dagger$ & 4 & \# \\
\hline A. effusus & CMXY 22,227 & $t$ & $\dagger$ & $t$ & I & $t$ & $t$ & \#\# \\
\hline A. flavus & CMXY 22,879 & $\dagger$ & $\dagger$ & $t$ & I & 2 & 4 & \#\# \\
\hline A. effusus & CMXY $27,48 I$ & $t$ & $\dagger$ & $t$ & I & 2 & 4 & \#\# \\
\hline A. flavus & CMXY 30, 197 & $\dagger$ & $t$ & $t$ & I & $\dagger$ & $\dagger$ & \#\# \\
\hline A. tubingensis & CMXY 27,207 & 16 & 16 & 16 & 2 & 16 & $\dagger$ & \#\# \\
\hline
\end{tabular}

Notes: "†" means the MIC $\leq$ epidemiological cutoff values; "\#” means the mutant site of cyp5IA gene was not be found; "\#\#” means the cyp5IA gene were not analyzed. Abbreviations: ITZ, itraconazole; VRZ, voriconazole; RVZ, ravuconazole; PSZ, posaconazole; ISZ, isavuconazole; AmB, amphotericin B; MIC, minimal inhibitory concentration.

Owing to the application of MLST, ${ }^{1,58}$ approximately $8-19 \%$ of isolates of Aspergillus spp. were recently identified as cryptic Aspergillus spp. in Spain and Portugal. ${ }^{27,59}$ Among those newly defined "cryptic" species, our samples contained four in two of the species complexes: $A$. tamarii and $A$. effusus in the A. flavus complex and $A$. tubingensis and A. awamori in the A. niger species complex. The frequencies of the cryptic species in our samples (15.3\%) are similar to those reported earlier. Interestingly, no cryptic species was identified in the A. fumigatus complex, which might be due to the limited number of clinical isolates. 
Currently, azoles, particularly VRZ, remain the preferred agents for treatment of IA. ${ }^{19,60}$ Our results suggested that most azoles (in particular PSZ) were highly active against Aspergillus spp. However, the reduced susceptibility to novel triazoles, such as RVZ and ISZ, was found in the $A$. niger complex. PSZ is the most potent drug against strains of $A$. fumigatus (GM MIC $0.187 \mu \mathrm{g} / \mathrm{mL}$ ) and the $A$. niger complex (GM MIC $0.416 \mu \mathrm{g} / \mathrm{mL}$ ), which was consistent with previous studies from China and Italy. ${ }^{17,30}$ The novel azoles ISZ and RVZ demonstrated a strong inhibitory activity against both A. fumigatus and A. flavus, similar to the results of previous studies from India and China. ${ }^{28,61}$ However, the reduced susceptibility (MIC $\geq 1 \mu \mathrm{g} / \mathrm{mL}$ ) of ISZ and RVZ among the majority isolates of the A. niger complex in our study was not consistent with the results of previous reports from Spain and the USA. ${ }^{36,62}$ In addition, our results also suggest that echinocandins (in particular AFG) are the potent drugs against clinical isolates of Aspergillus spp., which was similar to the previous studies from the USA, India and China. ${ }^{28,61,63}$ Therefore, our results support the current recommendation issued by the Infectious Diseases Society of America in using azoles as the primary treatment against Aspergillus spp. infections, ${ }^{19}$ in conjunction with echinocandins and/or polyenes if needed.

Since the first reported ITZ-resistant $A$. fumigatus isolate in $1997,{ }^{64}$ the isolates of azole-resistant $A$. fumigatus have been increasingly reported worldwide..$^{7,65,66}$ The amount of azole fungicide used in China is much higher than those used in European countries, ${ }^{26}$ which suggests a high selective pressure for environmental A. fumigatus. Several studies have indicated that the $\mathrm{TR}_{34} / \mathrm{L} 98 \mathrm{H}$ and $\mathrm{TR}_{34} / \mathrm{L} 98 \mathrm{H} / \mathrm{S} 297 \mathrm{~T} / \mathrm{F} 495 \mathrm{I}$ mutations were the predominant mutations in China. ${ }^{26,65,67}$ In 2015, three clinical isolates of A. fumigatus were found to harbor either the $\mathrm{TR}_{34} / \mathrm{L} 98 \mathrm{H} /$ $\mathrm{S} 297 \mathrm{~T} / \mathrm{F} 495 \mathrm{I}$ or the $\mathrm{TR}_{34} / \mathrm{L} 98 \mathrm{H}$ mutations in Fuzhou, Nanjing, and Shanghai. ${ }^{65}$ One study reported a worldwide clonal expansion of triazole-resistant isolates with the TR34/L98H mutations, while triazole-resistant isolates with the TR34/L98H/S297T/F495I mutation from China were genetically distinct from resistant isolates in other countries. ${ }^{26}$ Notably, the $\mathrm{TR}_{46} / \mathrm{Y} 121 \mathrm{~F} / \mathrm{T} 289 \mathrm{~A}$ mutation was found in one isolate of A. fumigatus (CMXY 13113). This isolate showed a high level of multi-azole resistance, similar to the result from the Netherlands in $2015 .{ }^{68}$ Studies so far have shown that isolates with the $\mathrm{TR}_{46} / \mathrm{Y} 121 \mathrm{~F} /$ T289A mutation are typically resistant to ITZ and VRZ but have variable susceptibility to PSZ. Such a result is different from that observed for strains with the $\mathrm{TR}_{34}$ /L98H mutation, which typically show ITZ resistance, but with variable susceptibility to VRZ and PSZ. ${ }^{69,70}$ To our knowledge, this is the first time the mutation has been identified within isolates of A. fumigatus in Shanghai, China. In addition, the F46Y, G89G, M172V, N248T and D255E mutations were found in two other isolates in our study, which also was observed in Australia and Netherlands. ${ }^{71,72}$ However, no cyp $51 \mathrm{~A}$ promoter or aminoacid sequence mutation was observed for the azole-resistant isolate CMXY25241. This result suggests that mutation(s) in gene(s) other than cyp51A was likely responsible. For example, a recent study reported that mutations in ATPbinding cassette $(\mathrm{ABC})$ or major facilitator superfamily (MFS) efflux pumps were associated with triazole resistance in A. fumigatus. $^{21}$ Our findings suggest diverse genetic backgrounds among the isolates of azole-resistant A. fumigatus from Shanghai, China. In addition, A. fumigatus isolates harboring the $\mathrm{TR}_{34} / \mathrm{L} 98 \mathrm{H}$ or $\mathrm{TR}_{46}$ /Y121F/T289A mutations were not only found in azolenaïve patients, but also found in soil and/or woody debris samples in New Zealand, India, Tanzania, and Africa, ${ }^{73}$ consistent with these mutations being originated from azole fungicide usage in the environment and agriculture.

\section{Conclusions}

Our study revealed a considerable diversity of Aspergillus spp. in lower respiratory tract of hospitalized patients in Shanghai, China. Although the frequency of azole resistance is relatively low (approximately 9\%), a number of strains showed resistance to multiple triazoles and even to multiple classes of antifungal drugs. The profiles of antifungal drug susceptibility provide important information for clinicians and local public health officials in determining the best treatment and prevention strategies. Our study emphasizes the need to not only identify all isolates of Aspergillus spp. at the species level, but also to perform antifungal susceptibility tests in clinical laboratories worldwide.

\section{Ethics Approval and Consent to Participate}

The protocol has been reviewed by the human research ethics committee of the Institutional Review Board (IRB) of Huashan Hospital Affiliated to Fudan University and since the project falls under the category observational study and all fungal strains were from residual samples used in clinical diagnosis or were strains from their subcultures, it has been 
determined they meet the criteria for exemption. The study was conducted in accordance with the Helsinki Declaration. Physicians working in the hospitals were consulted for the study to ensure the protections of life, health, dignity, privacy, and confidentiality of personal information of all study participants. After consultation with the IRB, formal ethical approval application was reviewed and waivered and written patient consent was deemed not required (Ethics Approval Number: KY2020-874).

\section{Acknowledgments}

We thank the Department of Dermatology, Shanghai Changzheng Hospital and Shanghai Huashan Hospital, for supporting this research. This work was supported by the National Natural Science Foundation of China (No. 81271762 and 81720108026), and Shanghai Science and Technology counsel projects of 16411961600 . The sponsor had no involvement in any of the stages from the study design to submission of the paper for publication.

\section{Author Contributions}

All authors made substantial contributions to conception and design, acquisition of data, or analysis and interpretation of data; took part in drafting the article or revising it critically for important intellectual content; agreed to submit to the current journal; gave final approval of the version to be published; and agree to be accountable for all aspects of the work.

\section{Disclosure}

The authors report no conflicts of interest in this work.

\section{References}

1. Samson RA, Visagie CM, Houbraken J, et al. Phylogeny, identification and nomenclature of the genus Aspergillus. Stud Mycol. 2014;78:141-173. doi:10.1016/j.simyco.2014.07.004

2. Park HS, Jun SC, Han KH, Hong SB, Yu JH. Diversity, application, and synthetic biology of industrially important Aspergillus fungi. $A d v$ Appl Microbiol. 2017;100:161-202.

3. Shishodia SK, Tiwari S, Shankar J. Resistance mechanism and proteins in Aspergillus species against antifungal agents. Mycology. 2019;10(3):151-165. doi:10.1080/21501203.2019.1574927

4. Sugui JA, Kwon-Chung KJ, Juvvadi PR, Latge JP, Steinbach WJ. Aspergillus fumigatus and related species. Cold Spring Harb Perspect Med. 2014;5(2):a019786. doi:10.1101/cshperspect.a019786

5. Yii AC, Koh MS, Lapperre TS, Tan GL, Chotirmall SH. The emergence of Aspergillus species in chronic respiratory disease. Front Biosci (Schol Ed). 2017;9:127-138. doi:10.2741/s477

6. Siqueira JP, Sutton DA, Garcia D, et al. Species diversity of Aspergillus section Versicolores in clinical samples and antifungal susceptibility. Fungal Biol. 2016;120(11):1458-1467. doi:10.1016/j. funbio.2016.02.006
7. Latge JP, Chamilos G. Aspergillus fumigatus and Aspergillosis in 2019. Clin Microbiol Rev. 2019;33(1).

8. Gago S, Denning DW, Bowyer P. Pathophysiological aspects of Aspergillus colonization in disease. Med Mycol. 2019;57 (Supplement_2):S219-S227. doi:10.1093/mmy/myy076

9. Ohara S, Tazawa Y, Tanai C, et al. Clinical characteristics of patients with Aspergillus species isolation from respiratory samples: comparison of chronic pulmonary aspergillosis and colonization. Respir Investig. 2016;54(2):92-97. doi:10.1016/j. resinv.2015.08.007

10. Dimopoulos G, Frantzeskaki F, Poulakou G, Armaganidis A. Invasive aspergillosis in the intensive care unit. Ann N Y Acad Sci. 2012;1272(1):31-39. doi:10.1111/j.1749-6632.2012.06805.x

11. Darling BA, Milder EA. Invasive aspergillosis. Pediat Rev. 2018;39 (9):476-478. doi:10.1542/pir.2017-0129

12. Giray B, Kocaogut E, Bu F, Haliki-Uztan A. Genotypic discrimination of Aspergillus fumigatus strain from related species within section fumigati. Ann Agric Environ Med. 2016;23(3):448-451.

13. Gautier M, Normand AC, Ranque S. Previously unknown species of Aspergillus. Clin Microbiol Infect. 2016;22(8):662-669. doi:10.1016/ j.cmi.2016.05.013

14. Verweij PE, Chowdhary A, Melchers WJ, Meis JF. Azole resistance in Aspergillus fumigatus: can we retain the clinical use of mold-active antifungal azoles? Clin Infect Dis. 2016;62(3):362-368. doi:10.1093/ $\mathrm{cid} / \mathrm{civ} 885$

15. Chowdhary A, Sharma C, Meis JF. Azole-resistant aspergillosis: epidemiology, molecular mechanisms, and treatment. J Infect Dis. 2017;216(suppl_3):S436-S444. doi:10.1093/infdis/jix210

16. Alastruey-Izquierdo A, Mellado E, Cuenca-Estrella M. Current section and species complex concepts in Aspergillus: recommendations for routine daily practice. Ann N Y Acad Sci. 2012;1273:18-24. doi:10.1111/j.1749-6632.2012.06822.x

17. Li Y, Wan Z, Liu W, Li R, Warnock DW. Identification and susceptibility of Aspergillus section nigri in china: prevalence of species and paradoxical growth in response to echinocandins. J Clin Microbiol. 2015;53(2):702-705. doi:10.1128/JCM.03233-14

18. Chen M, Xu Y, Hong N, et al. Epidemiology of fungal infections in China. Front Med. 2018;12(1):58-75.

19. Patterson TF, Thompson GR 3rd, Denning DW, et al. Practice guidelines for the diagnosis and management of aspergillosis: 2016 update by the infectious diseases society of America. Clin Infect Dis. 2016;63(4):e1-e60.

20. Kontoyiannis DP, Lewis RE. Antifungal drug resistance of pathogenic fungi. Lancet. 2002;359(9312):1135-1144. doi:10.1016/S01406736(02)08162-X

21. Pérez-Cantero A, López-Fernández L, Guarro J, Capilla J. Azole resistance mechanisms in Aspergillus: update and recent advances. Int $J$ Antimicrob Agents. 2020;55(1):105807. doi:10.1016/j. ijantimicag.2019.09.011

22. Vermeulen E, Maertens J, Schoemans H, Lagrou K. Azole-resistant Aspergillus fumigatus due to TR46/Y121F/T289A mutation emerging in Belgium, July 2012. Euro Surveill. 2012;17(48):20326.

23. Verweij PE, Mellado E, Melchers WJ. Multiple-triazole-resistant aspergillosis. N Engl J Med. 2007;356(14):1481-1483. doi:10.1056/ NEJMc061720

24. Meis JF, Chowdhary A, Rhodes JL, Fisher MC, Verweij PE. Clinical implications of globally emerging azole resistance in Aspergillus fumigatus. Philos Trans R Soc. 2016;371(1709):20150460.

25. Chen Y, Wang H, Lu Z, et al. Emergence of TR46/Y121F/T289A in an Aspergillus fumigatus isolate from a Chinese patient. Antimicrob Agents Chemother. 2015;59(11):7148-7150. doi:10.1128/AAC. 00887-15

26. Chen Y, Lu Z, Zhao J, et al. Epidemiology and molecular characterizations of azole resistance in clinical and environmental Aspergillus fumigatus isolates from China. Antimicrob Agents Chemother. 2016;60(10):5878-5884. doi:10.1128/AAC.01005-16 
27. Pinto E, Monteiro C, Maia M, et al. Aspergillus species and antifungals susceptibility in clinical setting in the North of Portugal: cryptic species and emerging azoles resistance in A. fumigatus. Front Microbiol. 2018;9:1656. doi:10.3389/fmicb.2018.01656

28. Deng S, Zhang L, Ji Y, et al. Triazole phenotypes and genotypic characterization of clinical Aspergillus fumigatus isolates in China Emerg Microbes Infect. 2017;6(12):e109. doi:10.1038/emi.2017.97

29. Bank S, Christensen K, Kristensen LH, Prag J. A cost-effectiveness analysis of identifying Fusobacterium necrophorum in throat swabs followed by antibiotic treatment to reduce the incidence of Lemierre's syndrome and peritonsillar abscesses. Eur J Clin Microbiol Infect Dis. 2013;32(1):71-78. doi:10.1007/s10096-012-1715-6

30. Iatta R, Nuccio F, Immediato D, et al. Species distribution and in vitro azole susceptibility of Aspergillus section Nigri isolates from clinical and environmental settings. J Clin Microbiol. 2016;54 (9):2365-2372. doi:10.1128/JCM.01075-16

31. Lackner M, Hagen F, Meis JF, et al. Susceptibility and diversity in the therapy-refractory genus scedosporium. Antimicrob Agents Chemother. 2014;58(10):5877-5885. doi:10.1128/AAC.03211-14

32. Peterson SW. Phylogenetic analysis of Aspergillus species using DNA sequences from four loci. Mycologia. 2008;100(2):205-226. doi:10.1080/15572536.2008.11832477

33. Siqueira JP, Sutton DA, Gene J, et al. Multilocus phylogeny and antifungal susceptibility of Aspergillus section Circumdati from clinical samples and description of A. pseudosclerotiorum sp. nov. J Clin Microbiol. 2017;55(3):947-958. doi:10.1128/JCM.02012-16

34. CLSI. 2017 Reference Method for Broth Dilution Antifungal Susceptibility Testing of fllamentous Fungi. CLSI Standard M38. 3rd ed. Wayne, PA: Clinical and Laboratory Standards Institute; 2017.

35. Espinel-Ingroff A, Fothergill A, Fuller J, Johnson E, Pelaez T, Turnidge J. Wild-type MIC distributions and epidemiological cutoff values for caspofungin and Aspergillus spp. for the CLSI broth microdilution method (M38-A2 document). Antimicrob Agents Chemother. 2011;55(6):2855-2859. doi:10.1128/AAC.01730-10

36. Espinel-Ingroff A, Chowdhary A, Gonzalez GM, et al. Multicenter study of isavuconazole MIC distributions and epidemiological cutoff values for Aspergillus spp. for the CLSI M38-A2 broth microdilution method. Antimicrob Agents Chemother. 2013;57(8):3823-3828. doi:10.1128/AAC.00636-13

37. Espinel-Ingroff A, Diekema DJ, Fothergill A, et al. Wild-type MIC distributions and epidemiological cutoff values for the triazoles and six Aspergillus spp. for the CLSI broth microdilution method (M38-A2 document). J Clin Microbiol. 2010;48(9):3251-3257. doi:10.1128/JCM.00536-10

38. Espinel-Ingroff A, Cuenca-Estrella M, Fothergill A, et al. Wild-type MIC distributions and epidemiological cutoff values for amphotericin $\mathrm{B}$ and Aspergillus spp. for the CLSI broth microdilution method (M38-A2 document). Antimicrob Agents Chemother. 2011;55 (11):5150-5154. doi:10.1128/AAC.00686-11

39. Ozmerdiven GE, Ak S, Ener B, et al. First determination of azole resistance in Aspergillus fumigatus strains carrying the TR34/L98H mutations in Turkey. J Infect Chemother. 2015;21(8):581-586. doi:10.1016/j.jiac.2015.04.012

40. Larkin MA, Blackshields G, Brown NP, et al. Clustal W and Clustal X version 2.0. Bioinformatics. 2007;23(21):2947-2948. doi:10.1093/ bioinformatics/btm404

41. Bongomin F, Gago S, Oladele RO, Denning DW. Global and multi-national prevalence of fungal diseases-estimate precision. J Fungi (Basel). 2017;3:4.

42. Walker TA, Lockhart SR, Beekmann SE, et al. Recognition of azole-resistant aspergillosis by physicians specializing in infectious diseases, United States. Emerg Infect Dis. 2018;24:1. doi:10.3201/ eid2401.170971

43. Ashu EE, Xu J. Strengthening the one health agenda: the role of molecular epidemiology in Aspergillus threat management. Genes. 2018;9(7). doi:10.3390/genes9070359
44. Cahill BC, Hibbs JR, Savik K, et al. Aspergillus airway colonization and invasive disease after lung transplantation. Chest. 1997;112 (5):1160-1164. doi:10.1378/chest.112.5.1160

45. Barberan J, Garcia-Perez FJ, Villena V, et al. Development of aspergillosis in a cohort of non-neutropenic, non-transplant patients colonised by Aspergillus spp. BMC Infect Dis. 2017;17(1):34. doi:10.1186/s12879-016-2143-5

46. Yan X, Li M, Jiang M, Zou LQ, Luo F, Jiang Y. Clinical characteristics of 45 patients with invasive pulmonary aspergillosis: retrospective analysis of 1711 lung cancer cases. Cancer. 2009;115 (21):5018-5025. doi:10.1002/cncr.24559

47. Liao Y, Chen M, Hartmann T, Yang RY, Liao WQ. Epidemiology of opportunistic invasive fungal infections in China: review of literature. Chin Med J. 2013;126(2):361-368.

48. Denning DW, Pleuvry A, Cole DC. Global burden of allergic bronchopulmonary aspergillosis with asthma and its complication chronic pulmonary aspergillosis in adults. Med Mycol. 2013;51(4):361-370. doi:10.3109/13693786.2012.738312

49. Greenberger PA, Bush RK, Demain JG, Luong A, Slavin RG, Knutsen AP. Allergic bronchopulmonary aspergillosis. J Allergy Clin Immunol Pract. 2014;2(6):703-708. doi:10.1016/j.jaip.2014.08.007

50. Fang ZG, Ouyang ZY, Liu P, Sun L, Wang XY. Airborne fungal community composition in indoor environments in Beijing. Huan Jing Ke Xue. 2013;34(5):2031-2037.

51. Hu J, Li N, Lv Y, Liu J, Xie J, Zhang H. Investigation on indoor air pollution and childhood allergies in households in Six Chinese Cities by subjective survey and field measurements. Int $J$ Environ Res Public Health. 2017;14:9. doi:10.3390/ijerph14090979

52. Gomez-Lopez A, Garcia-Effron G, Mellado E, Monzon A, Rodriguez-Tudela JL, Cuenca-Estrella M. In vitro activities of three licensed antifungal agents against spanish clinical isolates of Aspergillus spp. Antimicrob Agents Chemother. 2003;47 (10):3085-3088. doi:10.1128/AAC.47.10.3085-3088.2003

53. Rudramurthy SM, Paul RA, Chakrabarti A, Mouton JW, Meis JF. Invasive aspergillosis by Aspergillus flavus: epidemiology, diagnosis, antifungal resistance, and management. J Fungi (Basel). 2019;5:3.

54. Rudramurthy SM, Singh G, Hallur V, Verma S, Chakrabarti A. High fungal spore burden with predominance of Aspergillus in hospital air of a tertiary care hospital in Chandigarh. Indian J Med Microbiol. 2016;34(4):529-532. doi:10.4103/0255-0857.195359

55. Wang W, Zhao CY, Zhou JY, et al. Invasive pulmonary aspergillosis in patients with HBV-related liver failure. Eur J Clin Microbiol Infect Dis. 2011;30(5):661-667. doi:10.1007/s10096-010-1137-2

56. Severo LC, Geyer GR, Porto Nda S, Wagner MB, Londero AT. Pulmonary Aspergillus niger intracavitary colonization. Report of 23 cases and a review of the literature. Rev Iberoam Micol. 1997;14 (3):104-110.

57. Bongomin F, Batac CR, Richardson MD, Denning DW. A review of onychomycosis due to Aspergillus species. Mycopathologia. 2018;183(3):485-493. doi:10.1007/s11046-017-0222-9

58. Frisvad JC, Hubka V, Ezekiel CN, et al. Taxonomy of Aspergillus section Flavi and their production of aflatoxins, ochratoxins and other mycotoxins. Stud Mycol. 2019;93:1-63. doi:10.1016/j.simyco.2018. 06.001

59. Alastruey-Izquierdo A, Mellado E, Pelaez T, et al. Population-based survey of filamentous fungi and antifungal resistance in Spain (FILPOP Study). Antimicrob Agents Chemother. 2013;57 (7):3380-3387. doi:10.1128/AAC.00383-13

60. Verweij PE, Lestrade PPA, Melchers WJG, Meis JF. Azole resistance surveillance in Aspergillus fumigatus: beneficial or biased? J Antimicrob Chemother. 2016;71(8):2079-2082. doi:10.1093/jac/dkw259

61. Shivaprakash MR, Geertsen E, Chakrabarti A, Mouton JW, Meis JF. In vitro susceptibility of 188 clinical and environmental isolates of Aspergillus flavus for the new triazole isavuconazole and seven other antifungal drugs. Mycoses. 2011;54(5):e583-e589. doi:10.1111/ j.1439-0507.2010.01996.x 
62. Cuenca-Estrella M, Gomez-Lopez A, Mellado E, Garcia-Effron G, Monzon A, Rodriguez-Tudela JL. In vitro activity of ravuconazole against 923 clinical isolates of nondermatophyte filamentous fungi. Antimicrob Agents Chemother. 2005;49(12):5136-5138. doi:10.1128/ AAC.49.12.5136-5138.2005

63. Pfaller MA, Boyken L, Hollis RJ, et al. In vitro susceptibility of clinical isolates of Aspergillus spp. to anidulafungin, caspofungin, and micafungin: a head-to-head comparison using the CLSI M38-A2 broth microdilution method. J Clin Microbiol. 2009;47 (10):3323-3325. doi:10.1128/JCM.01155-09

64. Denning DW, Venkateswarlu K, Oakley KL, et al. Itraconazole resistance in Aspergillus fumigatus. Antimicrob Agents Chemother. 1997;41(6):1364-1368. doi:10.1128/AAC.41.6.1364

65. Liu M, Zeng R, Zhang L, et al. Multiple cyp51A-based mechanisms identified in azole-resistant isolates of Aspergillus fumigatus from China. Antimicrob Agents Chemother. 2015;59(7):4321-4325. doi:10.1128/AAC.00003-15

66. Korfanty GA, Teng L, Pum N, Xu J. Contemporary gene flow is a major force shaping the Aspergillus fumigatus population in Auckland, New Zealand. Mycopathologia. 2019;184(4):479-492. doi:10.1007/s11046-019-00361-8

67. Lockhart SR, Frade JP, Etienne KA, Pfaller MA, Diekema DJ, Balajee SA. Azole resistance in Aspergillus fumigatus isolates from the ARTEMIS global surveillance study is primarily due to the TR/ L98H mutation in the cyp51A gene. Antimicrob Agents Chemother. 2011;55(9):4465-4468. doi:10.1128/AAC.00185-11
68. van Ingen J, van der Lee HA, Rijs TA, et al. Azole, polyene and echinocandin MIC distributions for wild-type, TR34/L98H and TR46/Y121F/T289A Aspergillus fumigatus isolates in the Netherlands. J Antimicrob Chemother. 2015;70(1):178-181. doi:10. 1093/jac/dku364

69. Wiederhold NP, Patterson TF. Emergence of azole resistance in Aspergillus. Semin Respir Crit Care Med. 2015;36(5):673-680. doi:10.1055/s-0035-1562894

70. Hagiwara D, Watanabe A, Kamei K, Goldman GH. Epidemiological and genomic landscape of azole resistance mechanisms in Aspergillus fungi. Front Microbiol. 2016;7:1382. doi:10.3389/fmicb.2016.01382

71. Kidd SE, Goeman E, Meis JF, Slavin MA, Verweij PE. Multi-triazole -resistant Aspergillus fumigatus infections in Australia. Mycoses. 2015;58(6):350-355. doi:10.1111/myc.12324

72. Snelders E, Karawajczyk A, Schaftenaar G, Verweij PE, Melchers WJ. Azole resistance profile of amino acid changes in Aspergillus fumigatus CYP51A based on protein homology modeling. Antimicrob Agents Chemother. 2010;54(6):2425-2430. doi:10.1128/AAC.01599-09

73. Chowdhary A, Sharma C, van den Boom M, et al. Multi-azoleresistant Aspergillus fumigatus in the environment in Tanzania. J Antimicrob Chemother. 2014;69(11):2979-2983. doi:10.1093/jac/ dku259

\section{Publish your work in this journal}

Infection and Drug Resistance is an international, peer-reviewed openaccess journal that focuses on the optimal treatment of infection (bacterial, fungal and viral) and the development and institution of preventive strategies to minimize the development and spread of resistance. The journal is specifically concerned with the epidemiology of antibiotic resistance and the mechanisms of resistance development and diffusion in both hospitals and the community. The manuscript management system is completely online and includes a very quick and fair peerreview system, which is all easy to use. Visit http://www.dovepress.com/ testimonials.php to read real quotes from published authors. 